bioRxiv preprint doi: https://doi.org/10.1101/2021.05.22.445245; this version posted September 21, 2021. The copyright holder for this preprint (which was not certified by peer review) is the author/funder, who has granted bioRxiv a license to display the preprint in perpetuity. It is made available under aCC-BY 4.0 International license.

Running head: ENHANCED IEM

\title{
An enhanced inverted encoding model for neural reconstructions
}

\author{
Paul S. Scotti, Jiageng Chen, \& Julie D. Golomb \\ Department of Psychology, The Ohio State University, Columbus, Ohio, USA
}

Please address correspondence to:

Julie D. Golomb

The Ohio State University

Department of Psychology

1835 Neil Avenue

Columbus, $\mathrm{OH} 43210$

Email: golomb.9@osu.edu

Abstract word count: 150

Main text word count: 3732

Figures: 3 
ENHANCED IEM

\begin{abstract}
Inverted encoding models (IEMs) have recently become a popular method for investigating neural representations by reconstructing the contents of perception, attention, and memory from neuroimaging data. However, the standard IEM procedure can produce spurious results and interpretation issues. Here we present a novel modification to IEMs ("enhanced inverted encoding modeling," elEM) that addresses key issues inherent in the standard IEM procedure, improves the flexibility and interpretability of stimulus reconstructions, and provides trial-by-trial stimulus predictions and goodness-of-fit estimates. Our modifications are advantageous due to our decoding metric taking into account the choice of population-level tuning functions and employing a prediction error-based metric directly comparable across experiments. Our modifications also allow trial-by-trial confidence estimates independent of prediction error which can be used to threshold reconstructions and improve neural decoding performance and brain-behavior correlations. We validate the improved utility of elEM across three fMRI datasets and offer a Python package for easy implementation.
\end{abstract}


ENHANCED IEM

\section{Introduction}

A mental representation can be defined as the "systematic relationship between features of the natural world and the activity of neurons in the brain"1. An increasingly common approach to study mental representations using neuroimaging data is to employ encoding models, which describe this relationship computationally, typically by reducing the complexity of the input data with a set of functions that, when combined, roughly approximate the neural signal. Encoding and decoding models (aka voxelwise modeling or stimulus-model based modeling) have become a standard method for investigating neural representational spaces and predicting stimulus-specific information from brain activity ${ }^{2-4}$. The key advantages of such models over other computational approaches such as multivariate pattern classification or representational similarity analysis are typically touted as the following: (1) Encoding models can take inspiration from single-unit physiology by consisting of tuning functions in stimulus space (aka feature space), allowing both the maximally receptive feature and the precision/sensitivity of the tuning to be estimated across a population of neurons; (2) Encoding models (model that transforms stimuli into brain activity) are easily inverted into decoding models (model that transforms brain activity to stimuli) and can be applied to a wide range of stimuli (e.g., Gabor filters ${ }^{5,6}$, perceptual colors ${ }^{7}$, acoustic musical features ${ }^{8}$, human faces ${ }^{9}$ ); (3) The decoding model can predict, or reconstruct, novel stimuli or experimental conditions not used in the training of the model, with reconstructions offering interpretational advantages beyond classification-based decoding by yielding measures of activity across the full stimulus space and facilitating research questions exploring representational schemes for brain regions of interest ${ }^{74}$. 
ENHANCED IEM

The inverted encoding model (IEM) is one example of an encoding and decoding model that uses simple linear regression and a basis set representing the hypothesized population-level tuning functions, consisting of several channels that are modeled as cosines (or von Mises) equally separated across stimulus space (e.g., orientation, color, spatial location). Due to its simplicity, robust performance, and grounding in single-unit physiology principles, inverted encoding models have quickly risen to prominence in the cognitive neuroscience community ${ }^{10-39}$. The basic idea behind IEMs is that each channel in the basis set can be assigned a weight per voxel (we adopt fMRI nomenclature here, but IEMs can be applied to any modality like EEG, MEG, etc.) and hence a model can be trained to predict the activity of each voxel using the weights of each channel as predictors (i.e., the regressors in a linear regression). Then, this trained encoder is inverted such that it becomes a decoder capable of reconstructing a trial's stimulus when provided with a novel set of voxel activations (Figure $1 \mathrm{~A}$ ).

However, the standard implementation of IEM has some critical flaws and limitations. As discussed more below, it can produce misleading or difficult to interpret results, does not account for the shape of the basis channels (as recently pointed out in some high-profile debates in the literature $\left.{ }^{15,40}\right)$, and lacks a measure of decoding uncertainty. Compounding matters, various approaches exist for evaluating IEMs (Supplemental Figure 1) and often researchers report several decoding metrics due to ambiguity over which metric is best.

Here we present a novel modification to IEMs, subsequently referred to as "enhanced inverted encoding modeling" (eIEM), that improves the interpretability of 
ENHANCED IEM

stimulus reconstructions, addresses some key issues inherent in the standard IEM

procedure, and provides trial-by-trial stimulus predictions and goodness-of-fit estimates.

(a) Encoder:

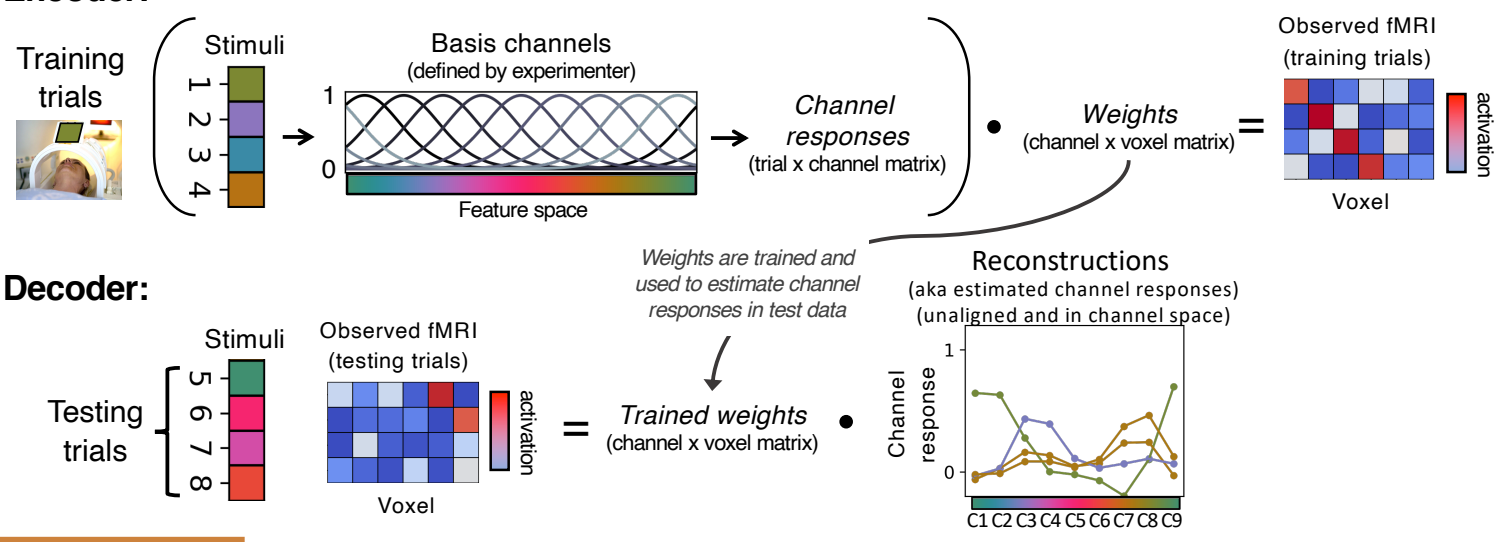

(b) Standard IEM

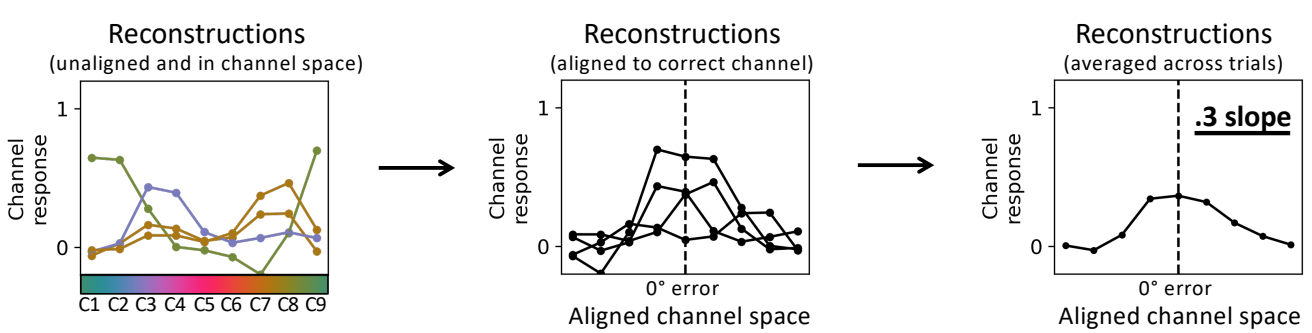

(c) elEM

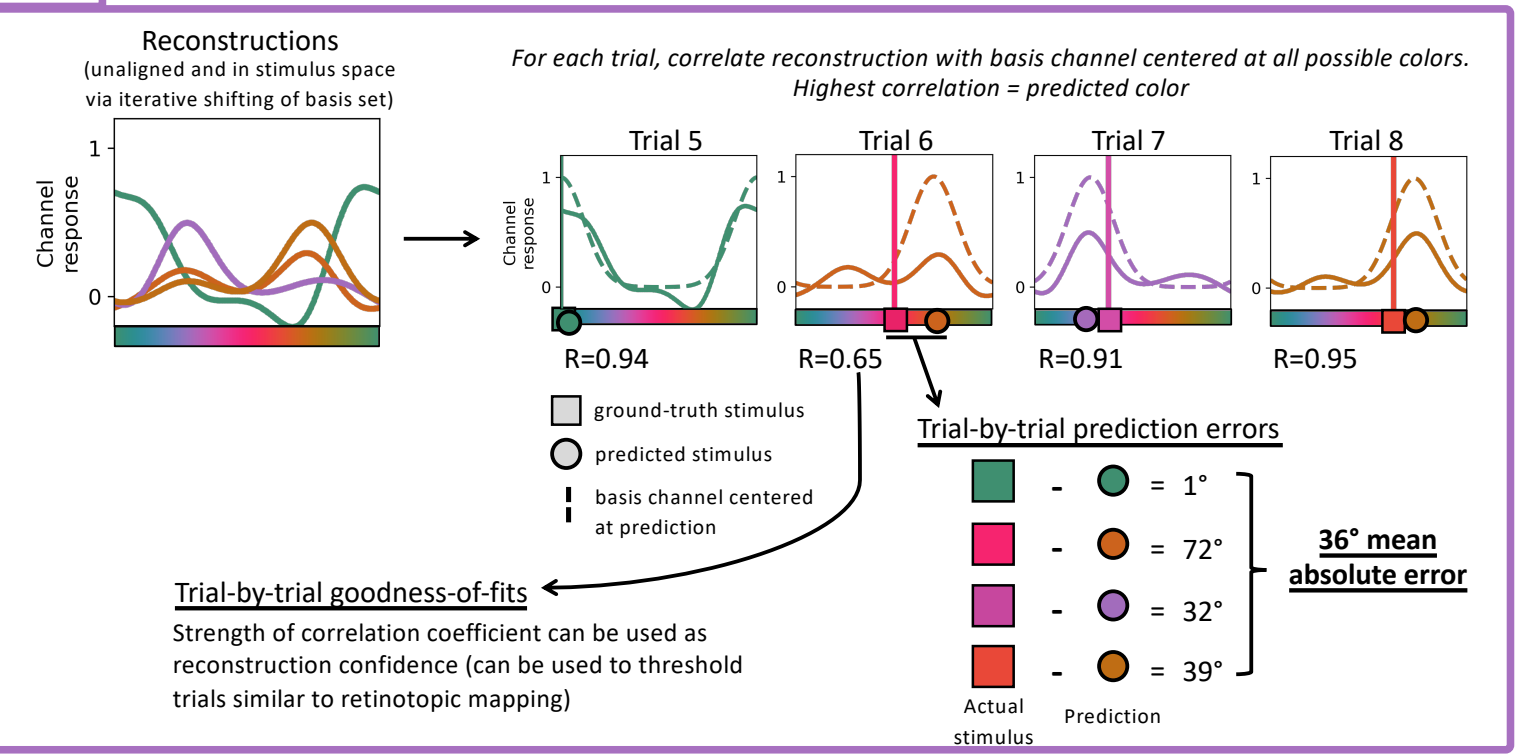


Figure 1. An overview of the steps involved in standard IEM and elEM using a toy example of an fMRI experiment reconstructing stimulus colors. (a) Basic encoder and decoder steps common to both standard and elEM. The prerequisites for implementing an IEM are the feature value of the stimulus for every trial (here 8 trials of colored squares), a trial-by-voxel matrix of brain activations for every voxel per trial (here simulated beta weights from a six-voxel brain region, and a basis set representing hypothesized population-level tuning functions; see Methods for details). The encoder models each voxel's response as the weighted sum of the channels. Given that the trial-by-voxel matrix and the basis set are already given, the weights matrix can be estimated via least-squares linear regression. Once the weights matrix is estimated from the training dataset, it can be inverted such that the encoder becomes a decoder for the test dataset. Now, instead of estimating the weights matrix via least-squares linear regression, the weights matrix and the trial-by-voxel matrix are given and the channel responses (i.e., reconstructions) are estimated. The resulting estimated channel responses, or simply reconstructions, is a trial-by-channel matrix where each trial has its own reconstruction composed of weighted cosines. For simplicity, this example shows the encoder trained on the first half of trials and the decoder used to predict the color of the remaining trials, but in most applications cross-validation should be used such that every trial may be decoded while avoiding circularity/double-dipping. (b) The standard procedure involves aligning and averaging reconstructions and measuring the result according to a variety of possible metrics (e.g., amplitude, slope; see Supplemental Figure 1). (c) elEM deviates from the standard procedure by evaluating prediction errors rather than an averaged reconstruction. We use iterative shifting of the basis set to allow channel space to equal stimulus space, correlate the reconstructions with the full set of basis channels to estimate each trial's predicted stimulus (and then average prediction error), and also estimate goodness-of-fits for each trial's reconstruction.

With elEM, the core encoder and decoder steps depicted in Figure 1A (i.e., leastsquares linear regression for estimating channel weights and responses, using a basis set of hypothesized population-level tuning functions) remain the same as standard IEM, but the encoding model is repeatedly fitted with slightly shifted basis sets such that subsequent reconstructions are in stimulus space rather than channel space (this iterative shifting of the basis set has been employed in a few previous papers ${ }^{18,38}$, but it is not common practice). Iterative shifting allows for more accurate reconstructions spanning the full stimulus space (allowing the remaining elEM steps to be optimally applied), and aids more generally in reducing interpretation flaws and bias associated with impoverished basis sets (see Supplemental Figure 2). 
More critically, the core difference of eIEM is that we evaluate reconstructions in terms of average prediction error instead of using the standard align-and-average reconstruction approach. In the standard IEM procedure, the trial-by-trial reconstructions are circularly shifted along the x-axis such that the "correct" channel (closest to the ground truth stimulus feature) is aligned to the center of the $\mathrm{x}$-axis (Figure 1B). The aligned reconstructions are then averaged together to result in a single reconstruction, which is then typically assessed using a number of possible metrics such as slope, amplitude, etc. As we show in the Results, this align-and-average approach is susceptible to information loss, outlier bias, and multiple interpretation issues.

To resolve this, elEM obtains trial-by-trial stimulus predictions using a correlation table approach (previously used in a small number of papers ${ }^{7,38}$ ) that adapts to the shape of the basis channel (Figure 1C). For each trial, a set of correlation coefficients is computed, each reflecting the correlation between that trial's reconstruction and a basis channel (i.e., "perfect reconstruction") centered at every integer in stimulus space (e.g., resulting in 360 correlation coefficients for a stimulus space ranging from $\left.0-359^{\circ}\right)$. The highest of these correlation coefficients is determined to be the best fit for that trial, and the predicted stimulus feature for that trial is simply the center of that best-fitting basis channel. The difference between the predicted and actual stimulus feature is then that trial's prediction error; this error is averaged across trials to obtain mean absolute error (MAE), which we recommend as a simple and easily interpretable metric. (Note that other error metrics are also possible in this framework, including signed error metrics if there is reason to expect asymmetric reconstructions.) 
Our final modification takes the trial-by-trial prediction approach described above one step further. In determining the best-fitting basis channel location, the center of that channel is taken as the stimulus prediction, but the goodness-of-fit (correlation coefficient) values themselves can also be optionally leveraged to estimate trial-by-trial confidence of predictions. Individual trials in a neuroimaging study can vary substantially in signal quality (driven by e.g., attentional fluctuations, alertness, head motion, scanner noise) but the standard IEM procedure does not incorporate uncertainty into decoding performance. The lack of uncertainty information has been noted in other contexts, with some recent alternatives to IEM proposed to incorporate uncertainty ${ }^{41,42}$. elEM easily and automatically produces a trial-by-trial measure of prediction uncertainty within the IEM framework itself. This enhancement adds substantial flexibility to the IEM procedure, e.g., allowing for thresholding reconstructions to potentially increase statistical power and performance, as we demonstrate in the Results.

In essence, the elEM approach that we present here is a combination of several modifications and improvements (some previously employed, some novel) on the standard IEM procedure. Researchers can easily implement elEM on their own through our publicly available Python package (see Methods). The value of elEM is primarily in terms of evaluating IEM results: using both real and simulated data, we show below that elEM is better than the standard IEM approach in terms of improved interpretability, flexibility, functionality, and robustness to methodological concerns.

\section{Results}


We first validate eIEM and demonstrate its practical advantages by implementing both standard IEM and eIEM on three real fMRI datasets ${ }^{13,18,43}$ spanning the research topics of perception, attention, and memory. We then use simulated reconstructions to illustrate additional methodological issues and limitations with standard IEM that eIEM addresses. See the Methods for information regarding each dataset and how data were processed.

\subsection{Validating elEM on real fMRI data}

First, as initial validation, we confirmed that across all three datasets elEM replicated the overall pattern of results obtained with the standard procedure (Figure 2). For consistency, we reanalyzed all datasets ourselves using a standard IEM procedure with slope as the decoding metric, even if the original paper did not employ this exact same procedure.

In the Perception dataset ${ }^{13}$, we used both techniques to decode the horizontal position of a stimulus in V1, V4, and IPS. The standard procedure revealed significant decoding performance in all 3 ROls, with the strongest decoding (greatest slope) in V1, followed by $\mathrm{V} 4$, and then IPS. eIEM replicated this pattern. In the Attention dataset ${ }^{43}$, we used both techniques to decode the attended orientation within a multi-item, multifeature stimulus array, in the same three ROls. The standard IEM procedure revealed significant decoding in V1 and V4, but not IPS. elEM again replicated this pattern. Finally, in the Memory dataset ${ }^{18}$, we used both techniques to decode the remembered orientation of a stimulus over two types of working memory delays: blank delay and distractor delay. With the standard procedure, the remembered orientation could be 
ENHANCED IEM

successfully decoded in V1, V4, and IPS, with significantly greater decoding in V1 and V4 during the blank delay compared to the distractor delay. With elEM, we replicated each of those results, with the additional finding of significantly greater decoding during the blank vs distractor delay in IPS. 
(a) Perception (Henderson, Vo, Chunharas, Sprague, \& Serences, 2019) Decode horizontal position

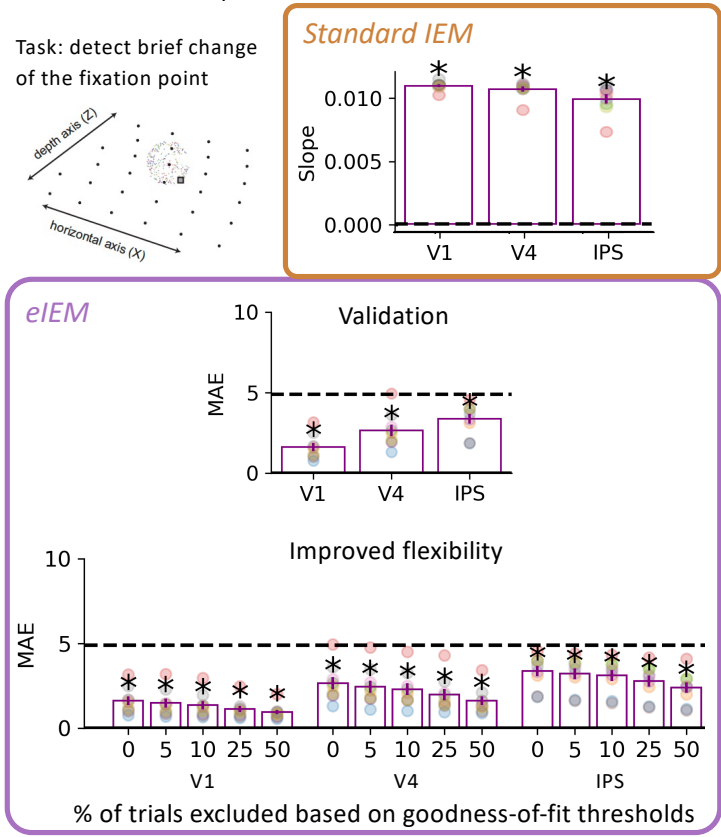

(c) Memory (Rademaker, Chunharas, \& Serences, 2019) Decode orientation during WM delay
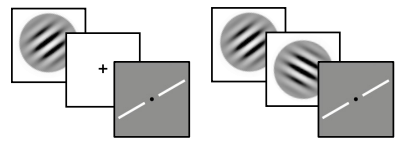
distractor grating
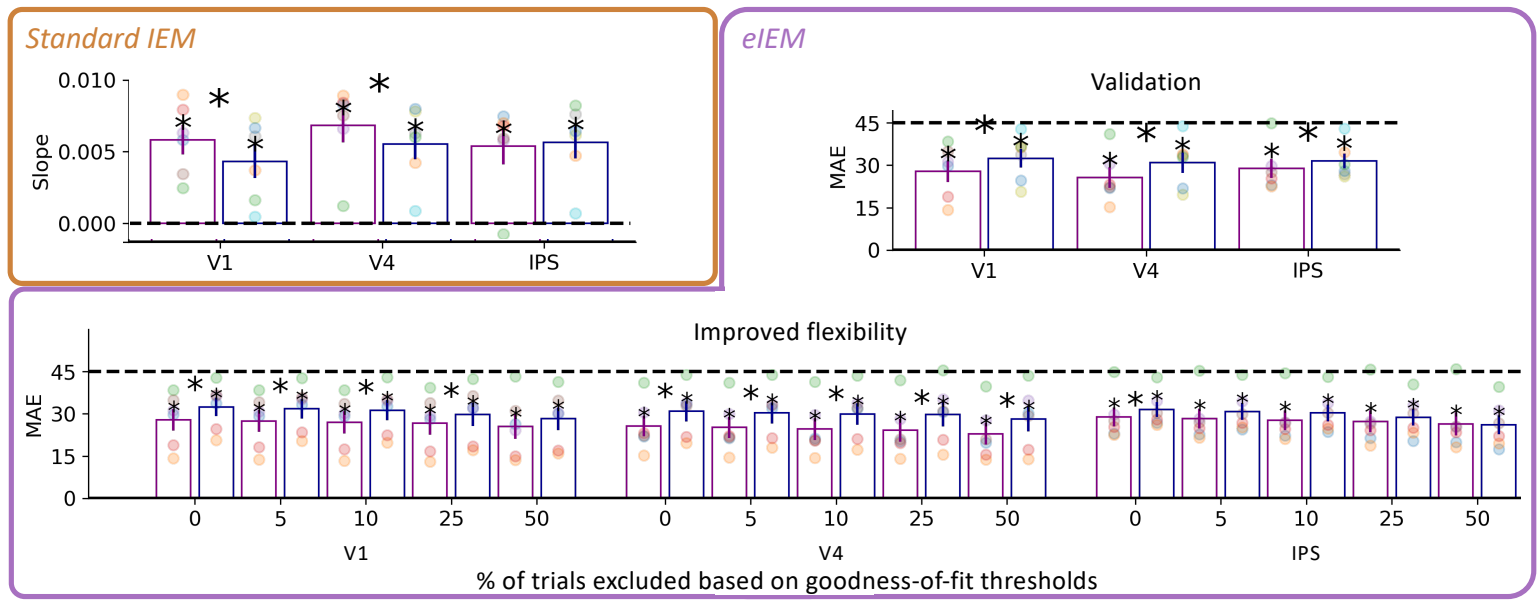

Figure 2. Results of the standard IEM procedure and our eIEM procedure across three real fMRI datasets spanning the topics of perception (a), attention (b), and memory (c). For each dataset, results are plotted obtained from the standard IEM procedure (orange boxes), elEM with full data (purple boxes, validation plots), and elEM using increasingly stringent cutoffs based on goodness-of-fit (purple boxes, improved flexibility plots). Bar plots depict the average slope from the standard procedure (higher values are better) and MAE from elEM (lower values are better) 
across subjects, with individual subjects overlaid as colored dots. For the Attention dataset, the brain-behavior correlation plot additionally plots the trial-by-trial correlation between absolute behavioral error and absolute decoding error for each ROI and goodness-of-fit threshold. Error bars depict standard error of the mean, dotted black lines represent chance decoding, and asterisks represent statistically significant decoding $(p<.05)$. Overall results show that conclusions are similar between the standard IEM and eIEM, but MAE is more interpretable (not based in arbitrary units) and not prone to methodological concerns discussed in the Introduction. In addition, each dataset showed that MAE consistently improved with increasing exclusion thresholds, demonstrating the flexibility of goodness-of-fit to exclude noisy trials. Increasing exclusion thresholds also appeared to strengthen brain-behavior correlations in the Attention dataset. See Supplementary Table 1 for test statistics and $p$ values.

\subsection{Demonstrating the improved interpretability and flexibility of elEMs}

Having validated our method across three diverse fMRI datasets, we next use these same datasets to illustrate the practical advantages of elEM.

Improved interpretability. elEM produces metrics that are easily interpretable and comparable across datasets due to decoding performance being measured in terms of prediction error. In contrast, the arbitrary units of the standard aligned-and-averaged reconstructions are not easily interpretable. For example, in the Memory dataset, the standard procedure results in an average slope of .006 for the blank delay condition and .004 for the distractor delay condition in V1 (or cosine fidelity values of .100 and .098 as reported in the original paper $^{18}$ ); elEM replicates this pattern, but now with a more interpretable and meaningful metric: orientation can be decoded with an average error of 27.9 degrees in the blank delay and 32.5 degrees in the distractor condition.

Incorporating trial-by-trial uncertainty. Next, we tested the flexibility of elEM to make use of the trial-by-trial goodness-of-fit information. The elEM approach produces a best-fitting stimulus prediction and associated goodness-of-fit value (correlation coefficient) for each trial. It is important to emphasize that the correlation coefficient reflects the degree to which the reconstruction matches the best-fitting basis channel, 
not the basis channel centered on the correct stimulus. In other words, this goodnessof-fit information is obtained independently and prior to any calculation of prediction error.

To test the impact of using goodness-of-fit information on decoding performance, we performed an analysis where we excluded trials with the lowest $5 \%, 10 \%, 25 \%$, and $50 \%$ of goodness-of-fit values (Figure 2). This resulted in visible improvements in MAE (i.e., smaller decoding error) with increasing exclusion thresholds for all three datasets (linear regression revealed significant negative slope in all cases except for IPS in the Attention dataset). Notably, in the Attention dataset, MAE improved with increasing thresholds in V1 and V4 (where decoding was significant in the unthresholded analysis) but not in IPS (where decoding was at chance in the unthresholded analysis). Thus, the goodness-of-fit information can be used to improve decoding performance when a brain region contains reliable information about a stimulus, but does not produce false positives in the absence of observable stimulus-specific brain activity.

This trial-by-trial prediction uncertainty information could be used in several ways. One suggestion we put forth is that goodness-of-fit can be used to threshold reconstructions, such that worse-fitting trials may be excluded from analysis. This principle is analogous to the phase-encoded retinotopic mapping and population receptive field modeling techniques, where a set of models spanning the full stimulus space is evaluated for every voxel, and the parameters of the best-fitting model are selected as that voxel's preferred stimulus, with the goodness-of-fit values then used to threshold the results ${ }^{44-46}$. Note that although r-squared is the more commonly used statistic for goodness-of-fit using regression, squaring the correlation coefficient is not 
ENHANCED IEM

preferred here because the sign of the correlation coefficient is informative (e.g., a perfectly inverted reconstruction should not be assigned equal confidence as a perfect reconstruction), so we recommend the use of the r-statistic.

Brain-behavior correlations. Finally, having trial-by-trial prediction error and goodness-of-fit values lends itself to analyses correlating neural measures with behavior. We demonstrate this in the Attention dataset (the Perception dataset did not collect behavioral responses, and in the Memory dataset behavioral performance was too close to ceiling $\left[\sim 3^{\circ}\right.$ avg. error]). In $\mathrm{V} 1$ and $\mathrm{V} 4$, we observed a significant correlation between a trial's behavioral error magnitude and neural prediction error. Moreover, the strength of these correlations increased with higher goodness-of-fit thresholds (Figure 2). We note that behavioral error itself did not noticeably change across these thresholds, suggesting that the goodness-of-fit information seemed to be reflecting noise at the level of the fMRI signal, not simply fluctuations in behavior or cognitive focus.

Altogether, these findings suggest that not only does elEM produce more interpretable and robust results, but the trialwise goodness-of-fit values offer increased flexibility to improve both neural decoding power and brain-behavior correlations.

\subsection{Additional methodological concerns addressed by elEM (simulations)}

The three real fMRI datasets analyzed above were useful validation cases because they contained robust findings (as may be more likely with published, publicly available datasets), allowing us to convey the improved interpretability and flexibility of elEM even in cases where the overall pattern of decoding is consistent with standard 
IEM. Crucially, however, there are also cases where we would expect elEM results to diverge from the standard IEM results, and to reflect more accurate decoding performance due to various methodological concerns inherent in the standard approach. Below we evaluate simulated reconstructions to illustrate specific methodological concerns and limitations of the standard IEM procedure that are addressed by elEM.

Standard IEM can produce misleading results. The standard procedure is susceptible to inappropriate decoding evaluations, largely due to the align-and-average step. Aligning and averaging across trial reconstructions loses information that is important for evaluating decoding performance and can be prone to heavy bias from outliers.

As depicted in Figure 3, averaging can obscure important information present in trial reconstructions. Panels $3 \mathrm{a}$ and $3 \mathrm{~b}$ would be interpreted identically according to standard IEM even though the first example shows every channel correctly predicted (i.e., predicting the correct stimulus feature with minimal error) and the second example shows every channel incorrectly predicted (large errors). elEM using MAE correctly identifies the first case as demonstrating superior decoding performance. The takeaway here is that averaging across prediction errors, and not across trial reconstructions, avoids the pitfall of interpreting Figures $3 a$ and $3 b$ as reflecting the same level of stimulus-specific brain signal despite clear support for Figure 3a demonstrating improved decoding on a trial-by-trial level. 

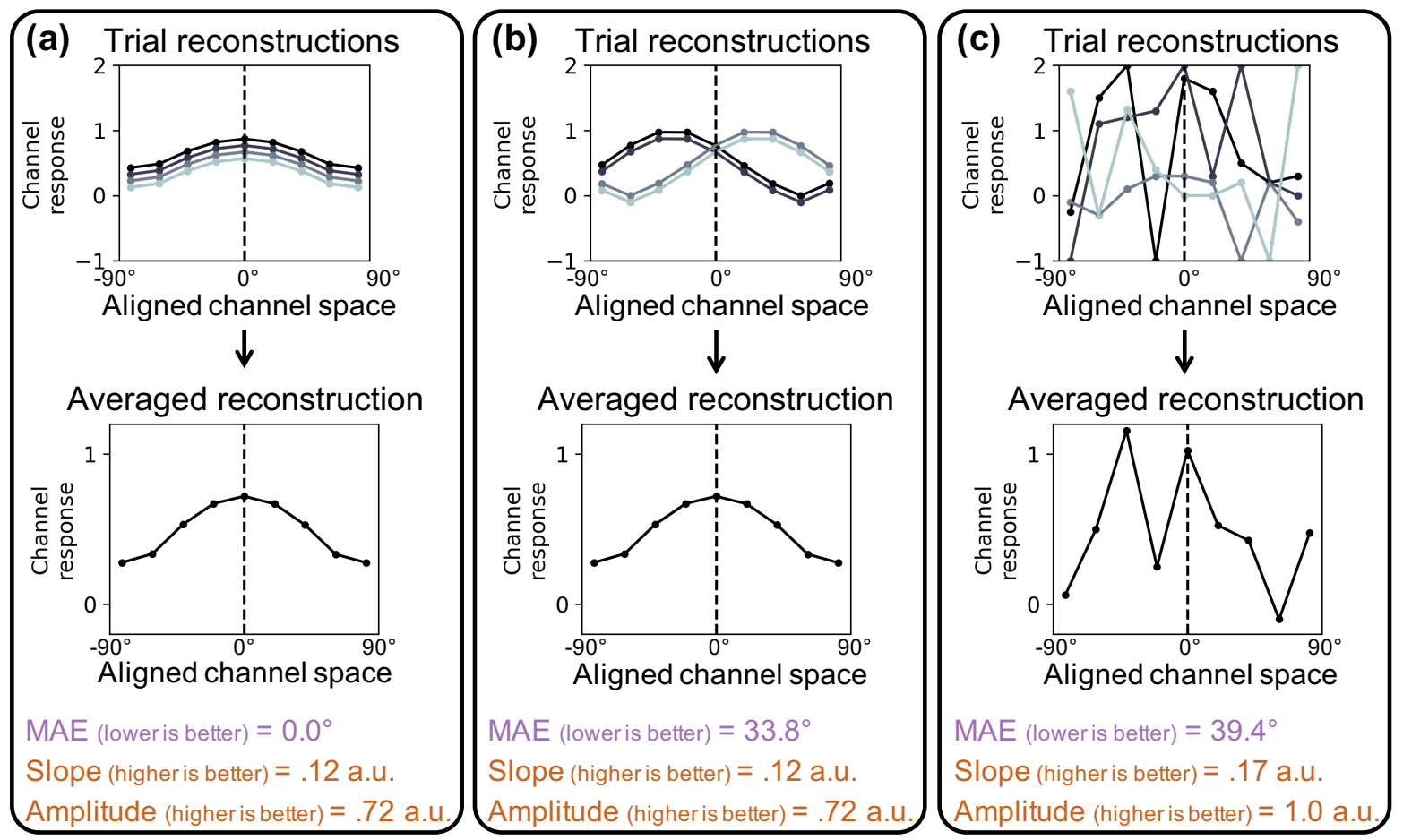

Figure 3. Simulated reconstructions depict some problems with the standard procedure of evaluating the aligned and averaged reconstruction and using a decoding metric that does not consider the shape of the basis channel or the variability of trial reconstructions. For each of the 3 simulated examples, the top row depicts four single-trial reconstructions, and the bottom row depicts the aligned-and-averaged reconstruction. The resulting values from the elEM (purple text) and standard IEM (orange text) metrics are shown below each example. In (a) each individual trial's reconstruction accurately predicts the correct channel (i.e., the correct stimulus feature), appropriately reflected in the averaged reconstruction. In (b) each individual trial's reconstruction predicts an incorrect channel. Averaging across trials leads to a misleading result, i.e., the standard approach would consider (b) to reflect the same level of decoding performance as (a). In (c) each individual trial's reconstruction is essentially noise, such that the averaged reconstruction results in a false peak around the aligned point; the standard procedure using align-and-average metrics results in spuriously superior decoding performance than both (a) and (b), with (c) having a higher amplitude, steeper slope, and narrower standard deviation when fit with a gaussian distribution. The elEM procedure, calculating MAE from trialwise prediction error, correctly concludes that case (a) shows the best decoding performance.

MAE is also less prone to bias from outlier reconstructions compared to any of the align-and-average metrics. In the standard procedure, a single outlier reconstruction can disproportionately bias the averaged reconstruction, potentially completely flipping the averaged reconstruction in the most extreme cases. In contrast, the influence of an outlier is naturally capped for elEM because prediction error (using MAE) is used as the 
decoding metric: Consider an experiment composed of 300 trials where 299 trials predict the correct stimulus and one trial predicts the stimulus $180^{\circ}$ away (assuming $360^{\circ}$ stimulus space); the outlier could only increase MAE by a maximum $0.6^{\circ}$.

Standard IEM does not account for the shape of the basis channels. IEMs produce reconstructions that depend on the choice of basis set ${ }^{15,40}$. The standard IEM procedure, however, does not take this observation into account. Supplemental Figure 2 illustrates one aspect of this issue that can be addressed with iterative shifting, but a more fundamental problem remains. That is, intuition - and standard practice - wrongly assume that a monotonic relationship exists between decoding metrics such as slope, amplitude, and bandwidth and a greater amount of stimulus-specific information in the brain signal. If the basis set consists of identical basis channels, then a perfect reconstruction returns the shape of the basis channel, and so it makes sense to compare the shape of the reconstruction to the shape of the basis channel to make predictions and evaluations. The correlation table metric employed in elEM automatically adjusts to consider the shape of the basis channel because it is the basis channel itself that is being used to obtain predictions, providing the most direct relationship between IEM performance and stimulus-specific brain signal.

Simply put, amplitude, slope, bandwidth, etc. are inferior metrics compared to the correlation table metric because they do not adapt to the choice of basis set. This is true even if a researcher were to skip the align-and-average step by evaluating reconstructions at the trial-by-trial level. For instance, using the amplitude metric, a higher amplitude at the aligned point is thought to reflect improved performance. If the basis channel ranges from 0 to 1 , a perfect reconstruction should have an amplitude of 
exactly 1 at the aligned point, but reconstructions can feasibly have amplitudes far greater than 1. Such a problem is demonstrated by comparing the simulated examples in Figure $3 \mathrm{C}$ versus 3A. Visually, it is clear that Figure $3 \mathrm{c}$ is a worse reconstruction than Figure 3a, but standard IEM metrics produce spuriously high values for this case.

It is possible to partially account for the shape of the basis channel by, for example, fitting the reconstruction with a gaussian distribution ${ }^{13}$. However, such fitting procedures may be problematic because such a procedure forces the reconstruction to appear to be a reasonable gaussian shape regardless of the data (e.g., fitting Figure 3c with a gaussian distribution would still lead to the same incorrect conclusion of superior decoding performance compared to Figure 3a).

\section{Discussion}

Inverted encoding modeling has become a popular method for predicting stimuli and investigating neural representations because of its robust performance, simplicity of linear modeling, ability to predict untrained classes, and grounding in single-unit physiology. Our new eIEM technique improves the flexibility and interpretability of results while fixing important methodological concerns surrounding the standard IEM procedure, namely how the standard procedure ignores trial-by-trial variability, does not account for the fact that a perfect reconstruction returns the basis channel, and cannot leverage uncertainty in its evaluations. The practical advantages of our method are made tangible by comparing the results of the standard IEM and elEM across three existing fMRI datasets (as well as simulated examples), highlighting the wide range of applications intended for elEMs. 
Importantly, our method can increase statistical power and IEM performance by leveraging uncertainty in model fits. Researchers have the flexibility to exclude trials with noisier reconstructions based on how similar in shape each reconstruction is to the basis channel at the predicted stimulus. Note that we do not prescribe a specific cutoff for determining goodness-of-fit thresholds in this paper, rather, we simply offer that such an approach is possible for improving IEM performance. For example, a researcher could a priori decide to more heavily weight trials with higher confidences or simply exclude the noisiest $20 \%$ of trials.

Our method also improves interpretability by evaluating reconstructions in terms of prediction error. For example, "V1 showed $10^{\circ}$ average prediction error and V4 showed $20^{\circ}$ average prediction error" is more interpretable than "V1 showed .02 amplitude and V4 showed .01 amplitude" because the latter is in arbitrary units, whereas MAE is in meaningful units. Further, unlike amplitude or slope, the magnitude of prediction error is not dependent on the choice of basis set and can be directly compared to other experiments using the same stimulus space.

We demonstrated the above two advantages using three fMRI datasets. Our validations further demonstrated how our IEM approach can be applied to both circular and non-circular stimulus spaces, is sensitive to variations in decoding performance across brain regions and experimental conditions, can be used to accurately decode the contents of perception, attention, and working memory, and can be used to meaningfully link neural reconstructions with behavior. Our modifications allowed for the decoding performance of each dataset to be directly compared to each other and demonstrated how uncertainty, measured via goodness-of-fit, can be leveraged to 
increase statistical power. Note that just because these three datasets produced consistent overall results (in terms of significance testing) across procedures does not ensure this will always be the case-for less reliable results, the methodological pitfalls of standard IEM become increasingly problematic, as shown in our evaluations of simulated reconstructions.

In this paper we have referred to IEMs as a specific kind of encoding and decoding model that involves simple linear regression with population-level tuning functions. There are more complex neuroimaging methods that can similarly be used to produce reconstructions via hypothesized tuning functions. For instance, Kay et al. ${ }^{5}$ decoded natural images from brain activity via voxel-level receptive field models that describe tuning functions across space, orientation, and spatial frequency. Naselaris et al. ${ }^{6}$ further produced Bayesian reconstructions of natural images via the combination of encoding models meant to estimate structural and semantic content. Van Bergen and colleagues $^{42,47,48}$ introduced models where voxels with similar tuning account for shared noise and which produce trial-by-trial probability distributions such that uncertainty can be obtained similarly to our procedure (although the researchers discuss this in terms of testing Bayesian theories of neural computation rather than trial thresholding). An advantage of elEMs is that improvements to the standard IEM approach are accomplished without sacrificing simplicity—the encoding model weights and the decoding model channel responses are simply estimated via least-squares estimation. Inverted encoding modeling has become increasingly popular in recent years, and yet the proper method for evaluating IEMs has become increasingly uncertain. Researchers often report IEM performance according to several metrics because of a 
lack of consensus regarding the "correct" way to evaluate reconstructions (see Supplementary Figure 1). Other decoding techniques in neuroimaging, such as support vector machines or neural networks, use the easily interpretable metric of classification performance (\% correct), but IEMs are typically evaluated in terms of arbitrary units that are abstracted away from the stimulus space they were intended to predict. We demonstrate a clear and practical advantage for evaluating reconstructions according to our method: researchers can increase their statistical power via thresholding, compare decoding performance across varying basis sets, evaluate performance in stimulus space, and obtain concrete stimulus predictions (with corresponding goodness-of-fits) for every trial rather than rely on a summary statistic based in arbitrary units. While there already exist approaches capable of addressing some of these concerns, our approach represents a suite of best practices that can be adopted by researchers in future work. Researchers can easily implement our procedure with one line of code using our accessible Python package (https://pypi.org/project/inverted-encoding; see Methods). 
ENHANCED IEM

\section{Funding}

This work was funded by the National Institutes of Health (R01-EY025648 to JDG) and the National Science Foundation (NSF DGE-1343012 to PSS, NSF BCS-1848939 to JDG).

\section{References}

1. Poldrack, R. A. The physics of representation. Synthese 1-19 (2020).

2. Naselaris, T., Kay, K. N., Nishimoto, S. \& Gallant, J. L. Encoding and decoding in fMRI. Neuroimage 56, 400-410 (2011).

3. Naselaris, T. \& Kay, K. N. Resolving ambiguities of MVPA using explicit models of representation. Trends Cogn. Sci. 19, 551-554 (2015).

4. Popov, V., Ostarek, M. \& Tenison, C. Practices and pitfalls in inferring neural representations. NeuroImage 174, 340-351 (2018).

5. Kay, K. N., Naselaris, T., Prenger, R. J. \& Gallant, J. L. Identifying natural images from human brain activity. Nature 452, 352-355 (2008).

6. Naselaris, T., Prenger, R. J., Kay, K. N., Oliver, M. \& Gallant, J. L. Bayesian reconstruction of natural images from human brain activity. Neuron 63, 902-915 (2009).

7. Brouwer, G. J. \& Heeger, D. J. Decoding and reconstructing color from responses in human visual cortex. J. Neurosci. 29, 13992-14003 (2009).

8. Casey, M., Thompson, J., Kang, O., Raizada, R. \& Wheatley, T. Population codes representing musical timbre for high-level fMRI categorization of music genres. in Machine Learning and Interpretation in Neuroimaging 34-41 (Springer, 2012). 
9. Lee, H. \& Kuhl, B. A. Reconstructing perceived and retrieved faces from activity patterns in lateral parietal cortex. J. Neurosci. 36, 6069-6082 (2016).

10. Ester, E. F., Sprague, T. C. \& Serences, J. T. Parietal and frontal cortex encode stimulusspecific mnemonic representations during visual working memory. Neuron $\mathbf{8 7}, 893-905$ (2015).

11. Ester, E. F., Anderson, D. E., Serences, J. T. \& Awh, E. A neural measure of precision in visual working memory. J. Cogn. Neurosci. 25, 754-761 (2013).

12. Foster, J. J., Bsales, E. M. \& Awh, E. Covert spatial attention speeds target individuation. J. Neurosci. 40, 2717-2726 (2020).

13. Henderson, M., Vo, V., Chunharas, C., Sprague, T. \& Serences, J. Multivariate analysis of BOLD activation patterns recovers graded depth representations in human visual and parietal cortex. Eneuro 6, (2019).

14. Kok, P., Rait, L. I. \& Turk-Browne, N. B. Content-based dissociation of hippocampal involvement in prediction. J. Cogn. Neurosci. 32, 527-545 (2020).

15. Liu, T., Cable, D. \& Gardner, J. L. Inverted encoding models of human population response conflate noise and neural tuning width. J. Neurosci. 38, 398-408 (2018).

16. Oh, B.-I., Kim, Y.-J. \& Kang, M.-S. Ensemble representations reveal distinct neural coding of visual working memory. Nat. Commun. 10, 1-12 (2019).

17. Yu, Q., Teng, C. \& Postle, B. R. Different states of priority recruit different neural representations in visual working memory. PLoS Biol. 18, e3000769 (2020).

18. Rademaker, R. L., Chunharas, C. \& Serences, J. T. Coexisting representations of sensory and mnemonic information in human visual cortex. Nat. Neurosci. 22, 1336-1344 (2019). 
19. Sprague, T. C. \& Serences, J. T. Attention modulates spatial priority maps in the human occipital, parietal and frontal cortices. Nat. Neurosci. 16, 1879-1887 (2013).

20. Sutterer, D. W., Foster, J. J., Adam, K. C., Vogel, E. K. \& Awh, E. Item-specific delay activity demonstrates concurrent storage of multiple active neural representations in working memory. PLoS Biol. 17, e3000239 (2019).

21. Cai, Y., Sheldon, A. D., Yu, Q. \& Postle, B. R. Overlapping and distinct contributions of stimulus location and of spatial context to nonspatial visual short-term memory. $J$. Neurophysiol. 121, 1222-1231 (2019).

22. Chen, N. et al. Sharpened cortical tuning and enhanced cortico-cortical communication contribute to the long-term neural mechanisms of visual motion perceptual learning. Neuroimage 115, 17-29 (2015).

23. Foster, J. J., Sutterer, D. W., Serences, J. T., Vogel, E. K. \& Awh, E. Alpha-band oscillations enable spatially and temporally resolved tracking of covert spatial attention. Psychol. Sci. 28, 929-941 (2017).

24. Garcia, J. O., Srinivasan, R. \& Serences, J. T. Near-real-time feature-selective modulations in human cortex. Curr. Biol. 23, 515-522 (2013).

25. Ho, T. et al. The optimality of sensory processing during the speed-accuracy tradeoff. $J$. Neurosci. 32, 7992-8003 (2012).

26. Kok, P. \& Turk-Browne, N. B. Associative prediction of visual shape in the hippocampus. $J$. Neurosci. 38, 6888-6899 (2018).

27. Lorenc, E. S., Sreenivasan, K. K., Nee, D. E., Vandenbroucke, A. R. \& D’Esposito, M. Flexible coding of visual working memory representations during distraction. J. Neurosci. 38, 5267-5276 (2018). 
28. Mostert, P. et al. Eye movement-related confounds in neural decoding of visual working memory representations. Eneuro 5, (2018).

29. Samaha, J., Sprague, T. C. \& Postle, B. R. Decoding and reconstructing the focus of spatial attention from the topography of alpha-band oscillations. J. Cogn. Neurosci. 28, 1090-1097 (2016).

30. Sprague, T. C., Ester, E. F. \& Serences, J. T. Reconstructions of information in visual spatial working memory degrade with memory load. Curr. Biol. 24, 2174-2180 (2014).

31. Sprague, T. C., Ester, E. F. \& Serences, J. T. Restoring latent visual working memory representations in human cortex. Neuron 91, 694-707 (2016).

32. Sprague, T. C., Itthipuripat, S., Vo, V. A. \& Serences, J. T. Dissociable signatures of visual salience and behavioral relevance across attentional priority maps in human cortex. $J$. Neurophysiol. 119, 2153-2165 (2018).

33. van Moorselaar, D. et al. Spatially selective alpha oscillations reveal moment-by-moment trade-offs between working memory and attention. J. Cogn. Neurosci. 30, 256-266 (2018).

34. Vo, V. A., Sprague, T. C. \& Serences, J. T. Spatial tuning shifts increase the discriminability and fidelity of population codes in visual cortex. J. Neurosci. 37, 3386-3401 (2017).

35. Yu, Q. \& Shim, W. M. Temporal-order-based attentional priority modulates mnemonic representations in parietal and frontal cortices. Cereb. Cortex 29, 3182-3192 (2019).

36. Kok, P., Mostert, P. \& De Lange, F. P. Prior expectations induce prestimulus sensory templates. Proc. Natl. Acad. Sci. 114, 10473-10478 (2017).

37. Tang, M. F., Arabzadeh, E. \& Mattingley, J. B. Forward modelling reveals dynamics of neural orientation tuning to unconscious visual stimuli during binocular rivalry. bioRxiv (2019) doi:10.1101/574905. 
38. Kim, I., Hong, S. W., Shevell, S. K. \& Shim, W. M. Neural representations of perceptual color experience in the human ventral visual pathway. Proc. Natl. Acad. Sci. 117, 1314513150 (2020).

39. Kok, P., Brouwer, G. J., van Gerven, M. A. \& de Lange, F. P. Prior expectations bias sensory representations in visual cortex. $J$. Neurosci. 33, 16275-16284 (2013).

40. Sprague, T. C., Boynton, G. M. \& Serences, J. T. The importance of considering model choices when interpreting results in computational neuroimaging. Eneuro 6, (2019).

41. Li, H.-H., Sprague, T. C., Yoo, A., Ma, W. J. \& Curtis, C. E. Joint representation of working memory and uncertainty in human cortex. bioRxiv (2021) doi:10.1101/2021.04.05.438511.

42. van Bergen, R. S. \& Jehee, J. F. TAFKAP: An improved method for probabilistic decoding of cortical activity. bioRxiv (2021) doi:10.1101/2021.03.04.433946.

43. Chen, J., Scotti, P. S., Dowd, E. W. \& Golomb, J. D. Neural Representations of Taskrelevant and Task-irrelevant Features of Attended Objects. bioRxiv (2021) doi:10.1101/2021.05.21.445168.

44. Dumoulin, S. O. \& Wandell, B. A. Population receptive field estimates in human visual cortex. Neuroimage 39, 647-660 (2008).

45. Engel, S. A., Glover, G. H. \& Wandell, B. A. Retinotopic organization in human visual cortex and the spatial precision of functional MRI. Cereb. Cortex N. Y. NY 1991 7, 181-192 (1997).

46. Sereno, M. I. et al. Borders of multiple visual areas in humans revealed by functional magnetic resonance imaging. Science 268, 889-893 (1995).

47. Van Bergen, R. S., Ma, W. J., Pratte, M. S. \& Jehee, J. F. Sensory uncertainty decoded from visual cortex predicts behavior. Nat. Neurosci. 18, 1728-1730 (2015). 
48. Van Bergen, R. S. \& Jehee, J. F. Modeling correlated noise is necessary to decode uncertainty. Neuroimage 180, 78-87 (2018).

49. Brouwer, G. J. \& Heeger, D. J. Cross-orientation suppression in human visual cortex. J. Neurophysiol. 106, 2108-2119 (2011).

50. Wandell, B. A., Dumoulin, S. O. \& Brewer, A. A. Visual field maps in human cortex. Neuron 56, 366-383 (2007).

51. Destrieux, C., Fischl, B., Dale, A. \& Halgren, E. Automatic parcellation of human cortical gyri and sulci using standard anatomical nomenclature. Neuroimage 53, 1-15 (2010).

52. Mumford, J. A., Turner, B. O., Ashby, F. G. \& Poldrack, R. A. Deconvolving BOLD activation in event-related designs for multivoxel pattern classification analyses. Neuroimage 59, 2636-2643 (2012). 


\section{Methods}

We performed analyses on both real and simulated data. For the real fMRI datasets, we used two publicly available published datasets ${ }^{13,18}$ and one unpublished dataset from our lab ${ }^{43}$. Note that we only analyzed a subset of the data from each dataset, analyzing one or two conditions across three brain regions for the sake of simplicity. The experimental paradigms and conditions / regions chosen are described more in each dataset's respective section below.

\section{Inverted encoding model procedures}

For all datasets, we performed a set of analyses using both the standard IEM and eIEM procedures (as depicted in Figure 1, with the exception that we used iterative shifting for both the standard IEM and elEM to facilitate more direct comparison). For both procedures, each basis channel was modeled as

$$
\cos \left((\theta-\mu) \frac{\pi}{\text { stimulus_range }}\right)^{\text {num_channels }-1}
$$

where $\theta$ is degrees in stimulus space, $\mu$ is the center of each channel, and stimulus_range is the range of stimulus space (e.g., $360^{\circ}$ hues on a color wheel). The reasoning behind raising cosines to the num_channels-1 is to make the tuning curves narrower and more comparable to physiological findings ${ }^{51}$. For the encoder, each voxel's response was modeled as the weighted sum of the channels such that the observed trial-by-voxel fMRI activation matrix is equal to the dot product of the basis set and the weight matrix, basis_set[trial_features, : ] channel_by_voxel_weights $=$ trial_by_voxel_activation 
where trial_features is the feature (e.g., orientation) of the stimulus and basis_set is the matrix of channels with shape (stimulus_range, num_channels).

Once the weights matrix is estimated from the training dataset, it is inverted such that the weights matrix and the trial-by-voxel matrix are given and the channel responses (i.e., reconstructions) are estimated.

trial_by_voxel_activation $\cdot$ channel_by_voxel_weights ${ }^{-1}=$ reconstructions

For all datasets, we used a basis set composed of nine equidistant channels each modeled as $\cos \left((\theta-\mu) \frac{\pi}{180}\right)^{8}$. We used 10-fold cross-validation, such that each iteration trained the model on $90 \%$ of the data and tested the model on the remaining $10 \%$, repeated such that all trials were at one point decoded as part of the testing set.

For the standard IEM procedure, we aligned and averaged the single trial reconstructions into an average reconstruction and calculated slope as a traditional decoding metric. For the elEM procedure, we calculated absolute prediction error for each trial via the correlation table metric and then calculated MAE. We performed these steps for each subject, ROI, and condition, and then we calculated the average slopes and MAEs across subjects.

For each condition and ROI, we assessed significance via permutation testing. Significance tests were one-sided and uncorrected, calculated by comparing the tstatistic calculated from the actual data against the permuted null distribution of $t-$ statistics (one t-statistic per each of 5,000 permutations). For elEMs, we also repeated this analysis pipeline using varying levels of goodness-of-fit thresholds. That is, we discarded a certain percent of trials based on the worst goodness-of-fits and then 
ENHANCED IEM

calculated MAE using the remaining trials. The full list of t-statistics and corresponding p-values for the tests performed in Figure 2 are displayed in Supplementary Table 1.

\section{Perception dataset: Henderson, Vo, Chunharas, Sprague, and Serences (2019)}

Data were obtained by downloading post-processed fMRI data associated with Henderson et al (2019) ${ }^{13}$, publicly available on OSF (https://osf.io/j7tpf/). In this experiment, nine participants attended to a central fixation while a sphere (multicolored flickering dots positioned on the shell of a 3D sphere with radius $3.4^{\circ}$ ) was presented at varying positions along the horizontal and depth axes (depth achieved through stereoscopic MR-compatible goggles). The task was to detect a brief luminance change of the fixation point. Participants completed between 7 and 21 runs, where each run of 36 trials began with a sphere presented for 3 s followed by a jittered intertrial interval (2$6 s)$. There were also runs where participants covertly attended to the sphere, but we did not include these runs in the analysis. We only reconstructed horizontal position for simplicity and because position-in-depth was only sampled across six unique locations (varied sampling across the entire stimulus space is more appropriate for inverted encoding models) whereas horizontal position was sampled across 36 unique locations (from $0.9^{\circ}$ to $9.8^{\circ}$ eccentricity in both directions, collapsing across position-in-depth). We analyzed V1, V4, and IPS regions of interest which were defined via retinotopic mapping protocols where participants viewed rotating wedges and bowtie stimuli ${ }^{52}$ while performing a covert attention task of detecting contrast dimming on a row of the checkerboard for the rotating wedge stimulus. We applied IEMs (following the procedures outlined earlier) to the post-processed data conducted by the authors of the 
ENHANCED IEM

original paper: Single-trial activation estimates consisted of averaged z-scored BOLD signal of the 3rd and 4th TRs following stimulus presentation. For more methods information, please refer to the original paper ${ }^{13}$.

\section{Attention dataset: Chen, Scotti, Dowd, \& Golomb (2021)}

Data were previously collected in our lab for another study ${ }^{43}$. In this experiment, seven participants completed a visual attention task. Each trial started with a central fixation cross. After $700 \mathrm{~ms}$, three circle outlines were displayed at equidistant locations surrounding the fixation cross for $200 \mathrm{~ms}$. One outline was thicker than the others, representing the spatial cue. Participants were instructed to covertly attend to the spatial cue location while maintaining fixation at the fixation cross. After $1100 \mathrm{~ms}$, three colored and oriented gratings were briefly displayed for $100 \mathrm{~ms}$, followed by a $200 \mathrm{~ms}$ mask and a continuous orientation report. Participants were instructed to report the orientation of the grating that appeared at the location of the spatial cue. There were also trials where participants were asked to shift attention to a different spatial location before the onset of the gratings, and entire runs where participants were asked to attend and report the color of the grating (instead of orientation), but we did not include these in our analysis. Participants completed at least 440 trials of each condition across multiple runs and sessions. We analyzed V1, V4, and IPS regions of interest: V1 and V4 were defined via retinotopic mapping protocols where participants viewed rotating wedges and bowtie stimuli ${ }^{52}$, while IPS was defined from the Destrieux atlas ${ }^{53}$ in Freesurfer (parcel labelled "S_intrapariet_and_P_trans"). To obtain single-trial neural activations for IEM, we modified a commonly used single-trial general linear model (GLM) approach ${ }^{42}$ to 
improve the model sensitivity and account for the large number of trials. Specifically, we conducted 40 GLMs per subject, where each GLM includes one regressor per run for one of the 40 trials in that run and one regressor per run for all the other remaining trials in that run. In this way, across the 40 GLMs, each trial in the experiment had an estimated single-trial beta weight. For more methods information, please refer to the original paper ${ }^{43}$.

\section{Memory dataset: Rademaker, Chunharas, and Serences (2019)}

Data were obtained by downloading post-processed fMRI data associated with Rademaker et al (2019) ${ }^{18}$, publicly available on OSF (https://osf.io/dkx6y). We reanalyzed Experiment 1, where six participants underwent a visual working memory task. For each trial, a cue indicating the distractor condition was shown for $1.4 \mathrm{~s}$, followed by a target grating shown for $.5 \mathrm{~s}$ where participants were instructed to memorize its orientation, followed by a $1 \mathrm{~s}$ blank delay, and then an $11 \mathrm{~s}$ delay where 3 possible distractor conditions were possible: blank delay, Fourier-filtered noise, or distractor grating of a pseudo-random orientation. Following an additional $1 \mathrm{~s}$ blank delay, participants had 3 s to report the orientation of the target grating, and finally a variable intertrial interval (3/5/8s). Each participant completed 108 trials per distractor condition. We only reconstructed the blank delay and distractor grating conditions for simplicity. We analyzed V1, V4, and IPS regions of interest which were defined via retinotopic mapping protocols where participants viewed rotating wedges and bowtie stimuli ${ }^{52}$. We applied IEMs to the post-processed data conducted by the authors of the original paper: Single-trial activation estimates consisted of averaged BOLD signal 
ENHANCED IEM

between 5.6-13.6s (7-17 TRs) after target onset. For more methods information, please refer to the original paper ${ }^{18}$.

\section{Simulated datasets}

We used Python to simulate fMRI data from hypothetical brain region of interests with arbitrarily chosen numbers of voxels, where each voxel's ground truth tuning function was the same shape as the basis channel. At least one voxel was always maximally receptive to each of the "presented" stimuli to ensure output of perfect reconstructions. For Figure 1, we injected random noise from a gaussian distribution into the trial by voxel matrix to produce noisier data as would be expected from a real fMRI experiment. Simulated fMRI data were subjected to the IEM procedures described above to depict the contents of Figure 1 and Supplemental Figure 2. For the analysis shown in Figure 3, we started with simulated reconstructions (manually defined channel responses selected to facilitate visualization of the different methodological pitfalls). For more details, the code used to produce these figures from their respective data are available on OSF (https://osf.io/et7m2/).

\section{Python package: inverted-encoding}

We have released the Python 3 package "inverted-encoding" on PyPi (https://pypi.org/project/inverted-encoding/) and GitHub (https://github.com/paulscotti/inverted_encoding) for easy implementation of our elEM procedure. The package contains two main functions, "IEM" and "permutation." 
For the "IEM" function, the only necessary inputs are an array of the stimulus features for every trial and a trial by voxel activations matrix (note: inputs other than voxels may be used for other modalities). The basis set can be specified as an optional parameter and will otherwise default to a basis set composed of nine equidistant channels each modeled as $\cos \left((\theta-\mu) \frac{\pi}{180}\right)^{8}$. The stimulus space defaults to a circular $0-179^{\circ}$ range but can be optionally set to other ranges. Non-circular stimulus spaces can be set by the Boolean parameter "is_circular." The IEM procedure defaults to a 10fold cross-validation procedure but can be optionally specified. The final outputs are an array of each trial's predicted stimulus and an array of each trial's corresponding goodness-of-fit. The user can then compute MAE themselves by averaging the (circular) absolute error between the predicted stimulus features and the actual stimulus features. The user can decide whether they want to threshold any trials using the provided goodness-of-fit values prior to calculating MAE.

For the "permutation" function, the only necessary input is an array of the actual stimulus features. For each iteration, the stimulus features are randomly shuffled and used as the predicted stimuli to compute the MAE. The function outputs a null distribution of MAE values for the user to compare against the MAE obtained from the "IEM" function. A more exact and computationally intensive method would be to rerun the entire IEM pipeline with shuffled stimulus labels on every iteration to obtain the null distribution. This can also be performed using our package by simply repeating the IEM function with a different shuffling of the stimulus features for every iteration. Our exploratory comparisons of null distributions obtained using both approaches across the three fMRI datasets discussed in the main text yielded no obvious differences. 


\section{Data availability}

The Perception datase ${ }^{13}$ is publicly available on OSF (https://osf.io/j7tpf/). The Memory dataset ${ }^{18}$ is publicly available on OSF (https://osf.io/dkx6y). The Attention dataset ${ }^{43}$ will be made publicly available upon final publication of the original study; researchers may contact the authors in the meantime.

\section{Code availability}

Code to implement elEM is available as a Python package (https://pypi.org/project/inverted-encoding). Code to reproduce Figures 1, 3, and Supplementary Figure 2 using simulated data are available on OSF (https://osf.io/et7m2/). 
ENHANCED IEM

\section{Supplemental Information}

An enhanced inverted encoding model for neural reconstructions

Paul S. Scotti, Jiageng Chen, \& Julie D. Golomb 
ENHANCED IEM

\section{Table of Contents}

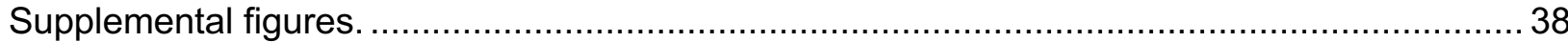

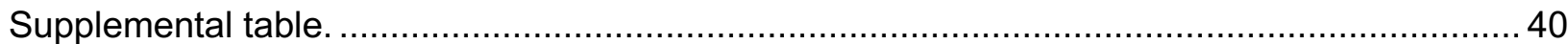

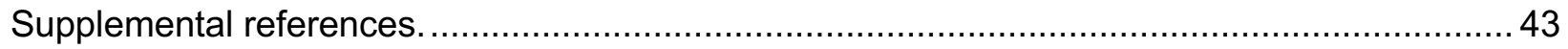




\section{Supplemental figures.}

Metrics used to evaluate inverted encoding models Standard approach (aligned and averaged reconstruction) $\frac{7}{3}$

- Amplitude: Measure the height of the aligned $x$-axis location of the reconstruction.

- Slope: Fold the reconstruction in half (vertically), average the two halves and then take the resulting slope.

- Fit amplitude: Fit a gaussian distribution to the reconstruction and then measure the resulting amplitude at the aligned $x$-axis location.

- Fit bandwidth: Fit a gaussian distribution to the reconstruction and then measure the resulting standard deviation.

- Cosine fidelity: Multiply the reconstruction by a cosine (with height ranging from -1 to 1 ) and then average the amplitudes across all points.

Non-standard approach (individual trial estimates)

- Maximum point: The point in stimulus space with the highest amplitude becomes that trial's predicted stimulus.

- Correlation table: For each trial, correlate the reconstruction with a basis channel centered at every integer in stimulus space. Select the channel with the largest correlation coefficient. Predicted stimulus $=$ center of this channel.
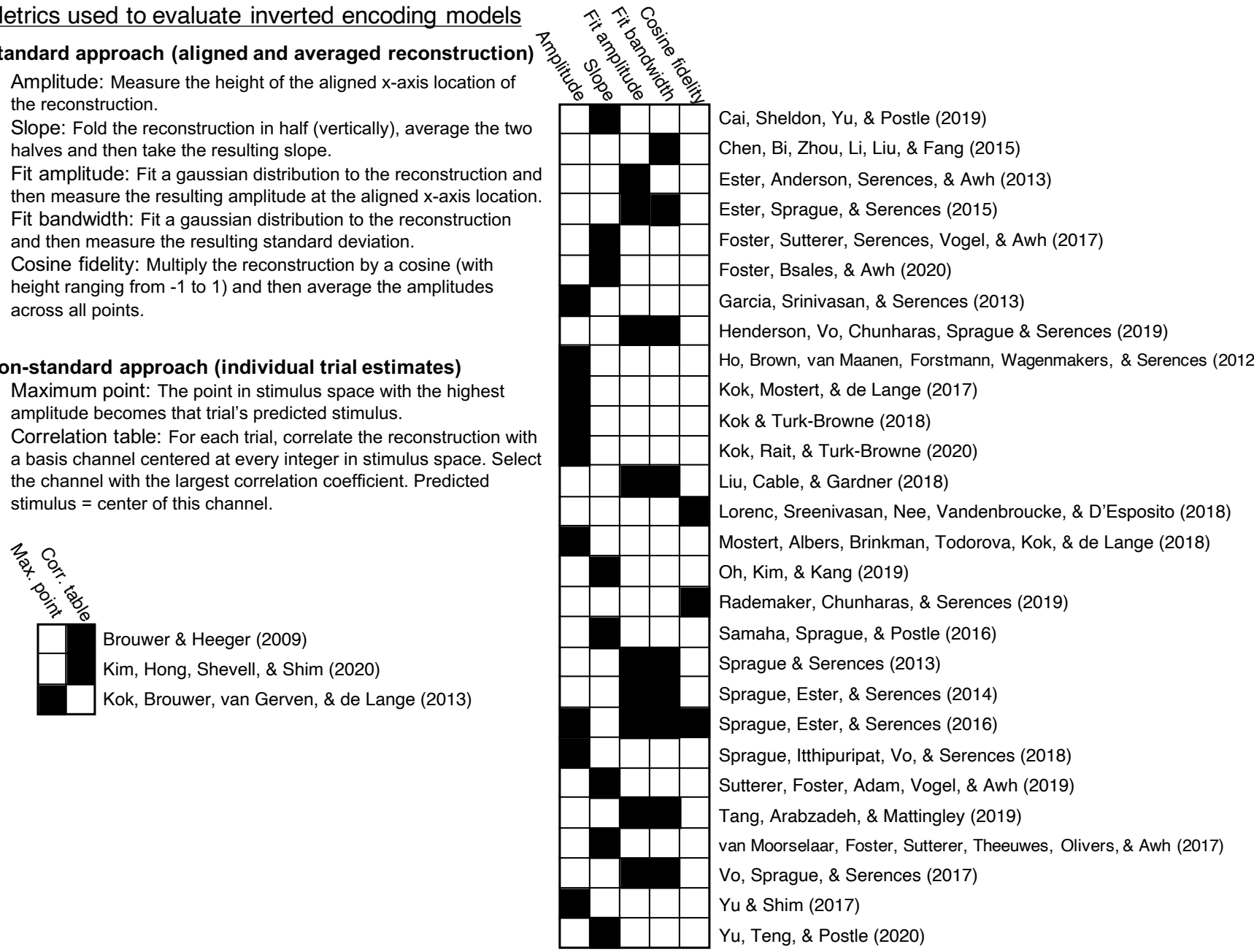

Supplemental Figure 1. Summary of metrics used to evaluate IEM reconstructions in a sampling of published papers ${ }^{1-30}$. Note that the methodological concerns raised in the Results apply to the metrics labeled under the standard approach, although our proposed modifications pose improvements over typical applications of "maximum point" and "correlation table" approaches as well. The non-standard approaches can be quantified with a single value (like the standard approach metrics) by taking the mean absolute error between predicted and actual stimuli. 
(a)

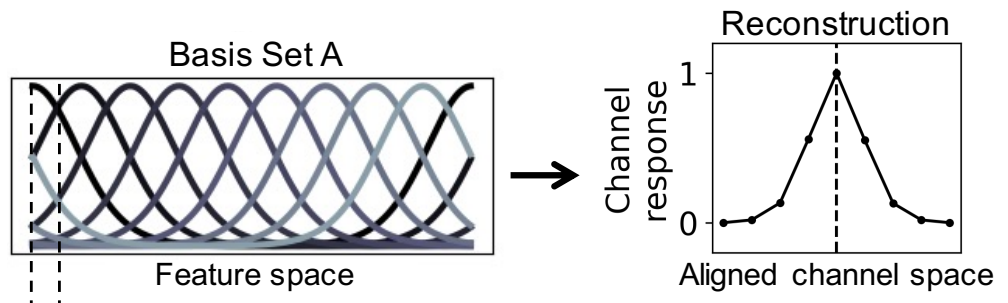

(b)

$20^{\circ}$ shift Basis Set B

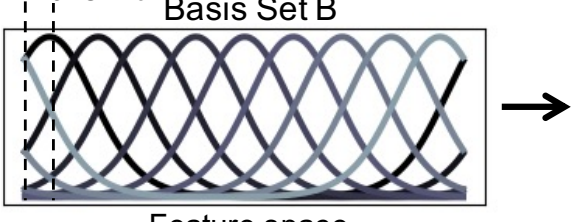

Feature space

(c)

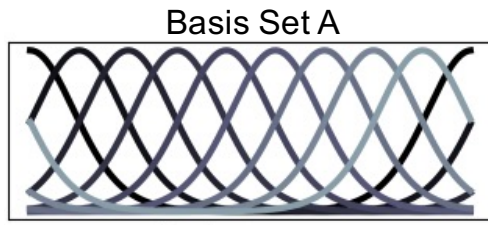

Feature space

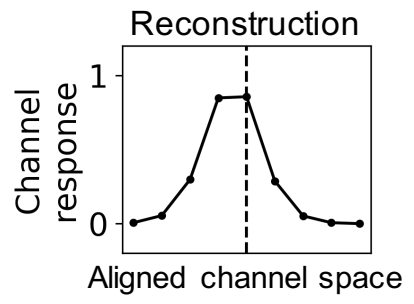

Basis Set B

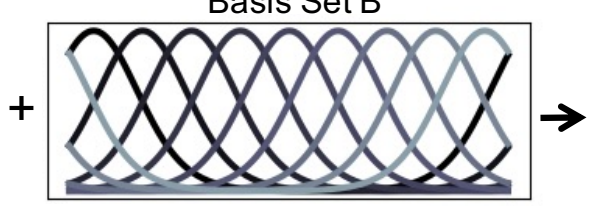

Feature space

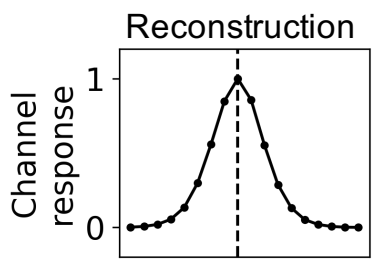

Aligned channel space

Supplemental Figure 2. Simulation depicting iterative shifting and its benefits for IEM reconstructions. The simulated neuroimaging data (trial by voxel activations) are identical for all three cases shown, and the underlying voxel tuning functions for this hypothetical brain region are known (simulated ground truth). The trial by voxel activations were constructed to reflect perfect (zero noise) information with identical train and test sets, such that the resulting reconstructions should also be perfect. (a) Basis set happens to perfectly reflect the underlying voxel tuning functions (simulated ground truth). (b) Reconstruction of the same data, now with a slightly altered basis set (channel means circularly shifted by $20^{\circ}$ ). Note that in a real experiment, the ground truth actual voxel tuning functions are not known, so the experimenter's arbitrary choice of channel centers could substantially alter resulting reconstructions, even if the same signal is present in all cases. This could result in misleading conclusions using the standard IEM technique. (c) By combining the results of both basis sets, the channel space changes from num_channels to num_channels*2, leading to a fuller reconstruction. This is the principle of iterative shifting: In elEM, we repeatedly fit the encoding model with every possible (circular) shift of the basis set (i.e. $1^{\circ}$ shifts) and then combine all of these iterations together. This produces a fuller reconstruction that is no longer impoverished by a limited number of num_channels points (i.e., the range of channel space becomes equal to the range of stimulus space) and allows the correlation table metric to be optimally applied. This iterative shifting procedure also aids more generally in producing more interpretable and less biased reconstructions, as it allows our decoding model to be capable of predicting any possible feature in stimulus space (that is, not solely the stimuli that are located at the centers of the basis channels). Note that iterative shifting does not change the fact that different basis sets result in different reconstructions, rather, it simply allows for the most accurate reconstruction given a set number of channels with defined bandwidths. 
ENHANCED IEM

Supplemental table.

\begin{tabular}{|c|c|c|c|c|}
\hline Dataset & Procedure & Test description & T-stat & $\mathrm{P}-$ \\
\hline \multirow[t]{18}{*}{ Perception } & IEM & $\mathrm{V} 1$ & 106.06 & .000 \\
\hline & & V4 & 53.33 & .000 \\
\hline & & IPS & 27.70 & .000 \\
\hline & elEM & V1 & -13.58 & .000 \\
\hline & & V4 & -6.61 & .000 \\
\hline & & IPS & -5.12 & .000 \\
\hline & & V1 (5\% excluded) & -13.73 & .000 \\
\hline & & V1 (10\% excluded) & -15.79 & .000 \\
\hline & & V1 (25\% excluded) & -22.06 & .000 \\
\hline & & V1 (50\% excluded) & -29.65 & .000 \\
\hline & & V4 (5\% excluded) & -7.11 & .000 \\
\hline & & V4 (10\% excluded) & -7.97 & .000 \\
\hline & & V4 (25\% excluded) & -9.18 & .000 \\
\hline & & V4 (50\% excluded) & -13.63 & .000 \\
\hline & & IPS (5\% excluded) & -5.54 & .000 \\
\hline & & IPS (10\% excluded) & -5.86 & .000 \\
\hline & & IPS (25\% excluded) & -6.78 & .000 \\
\hline & & IPS (50\% excluded) & -8.54 & .000 \\
\hline \multirow[t]{22}{*}{ Attention } & IEM & $\mathrm{V} 1$ & 16.02 & .000 \\
\hline & & V4 & 7.65 & .001 \\
\hline & & IPS & -0.16 & .601 \\
\hline & elEM & V1 & -9.93 & .000 \\
\hline & & V4 & -7.29 & .000 \\
\hline & & IPS & -0.11 & .535 \\
\hline & & V1 (5\% excluded) & -9.64 & .000 \\
\hline & & V1 (10\% excluded) & -9.79 & .000 \\
\hline & & V1 (25\% excluded) & -10.08 & .000 \\
\hline & & V1 (50\% excluded) & -11.49 & .000 \\
\hline & & V4 (5\% excluded) & -7.36 & .000 \\
\hline & & V4 (10\% excluded) & -7.42 & .000 \\
\hline & & V4 (25\% excluded) & -7.88 & .000 \\
\hline & & V4 (50\% excluded) & -7.86 & .000 \\
\hline & & IPS (5\% excluded) & -1.15 & .208 \\
\hline & & IPS (10\% excluded) & -1.06 & .234 \\
\hline & & IPS ( $25 \%$ excluded) & -0.87 & .281 \\
\hline & & IPS (50\% excluded) & 0.98 & .842 \\
\hline & elEM - brain-behavior & V1 & 7.02 & .000 \\
\hline & & V4 & 5.18 & .002 \\
\hline & & IPS & -1.06 & .330 \\
\hline & & V1 (5\% excluded) & 6.50 & .001 \\
\hline
\end{tabular}




\begin{tabular}{|c|c|c|c|c|}
\hline & & V1 (10\% excluded) & 6.30 & .001 \\
\hline & & V1 (25\% excluded) & 7.25 & .000 \\
\hline & & V1 (50\% excluded) & 6.69 & .001 \\
\hline & & V4 (5\% excluded) & 4.48 & .003 \\
\hline & & V4 (10\% excluded) & 4.81 & .003 \\
\hline & & V4 (25\% excluded) & 5.18 & .002 \\
\hline & & V4 (50\% excluded) & 7.03 & .000 \\
\hline & & IPS (5\% excluded) & -0.85 & .427 \\
\hline & & IPS (10\% excluded) & -1.38 & .218 \\
\hline & & IPS ( $25 \%$ excluded) & -0.70 & .508 \\
\hline & & IPS (50\% excluded) & -0.41 & .693 \\
\hline \multirow[t]{33}{*}{ Memory } & IEM - blank delay & $\mathrm{V} 1$ & 6.21 & .001 \\
\hline & & V4 & 6.38 & .002 \\
\hline & & IPS & 4.70 & .004 \\
\hline & IEM - distractor delay & V1 & 4.07 & .008 \\
\hline & & V4 & 5.55 & .001 \\
\hline & & IPS & 5.57 & .001 \\
\hline & IEM - blank vs. distractor & V1 & 4.18 & .013 \\
\hline & & V4 & 4.18 & .011 \\
\hline & & IPS & -0.53 & .659 \\
\hline & elEM - blank delay & V1 & -4.88 & .006 \\
\hline & & V4 & -5.79 & .003 \\
\hline & & IPS & -5.22 & .005 \\
\hline & & V1 (5\% excluded) & -4.94 & .006 \\
\hline & & V1 (10\% excluded) & -4.99 & .006 \\
\hline & & V1 (25\% excluded) & -4.86 & .006 \\
\hline & & V1 (50\% excluded) & -4.75 & .007 \\
\hline & & V4 (5\% excluded) & -5.77 & .003 \\
\hline & & V4 (10\% excluded) & -5.76 & .003 \\
\hline & & V4 (25\% excluded) & -5.70 & .003 \\
\hline & & V4 (50\% excluded) & -6.21 & .002 \\
\hline & & IPS (5\% excluded) & -5.15 & .005 \\
\hline & & IPS (10\% excluded) & -5.43 & .004 \\
\hline & & IPS ( $25 \%$ excluded) & -4.95 & .006 \\
\hline & & IPS (50\% excluded) & -5.00 & .006 \\
\hline & elEM - distractor delay & $\mathrm{V} 1$ & -4.07 & .012 \\
\hline & & V4 & -4.26 & .010 \\
\hline & & IPS & -5.65 & .002 \\
\hline & & V1 (5\% excluded) & -4.26 & .010 \\
\hline & & V1 (10\% excluded) & -4.25 & .010 \\
\hline & & V1 (25\% excluded) & -4.08 & .012 \\
\hline & & V1 (50\% excluded) & -4.50 & .008 \\
\hline & & V4 (5\% excluded) & -4.25 & .010 \\
\hline & & V4 (10\% excluded) & -4.30 & .010 \\
\hline
\end{tabular}


ENHANCED IEM

\begin{tabular}{|l|c|c|c|c|}
\hline & & V4 (25\% excluded) & -3.65 & .019 \\
\hline & & V4 (50\% excluded) & -4.09 & .012 \\
\hline & & IPS (5\% excluded) & -5.18 & .005 \\
\hline & & IPS (10\% excluded) & -5.35 & .004 \\
\hline & & IPS (25\% excluded) & -5.92 & .002 \\
\hline & & IPS (50\% excluded) & -6.11 & .001 \\
\hline & elEM - blank vs. distractor & V1 & -7.55 & .000 \\
\hline & & V4 & -2.85 & .049 \\
\hline & & IPS & -3.03 & .041 \\
\hline & & V1 (5\% excluded) & -7.14 & .001 \\
\hline & & V1 (10\% excluded) & -7.08 & .001 \\
\hline & & V1 (50\% excluded) & -2.73 & .056 \\
\hline & & V4 (5\% excluded) & -2.89 & .048 \\
\hline & & V4 (10\% excluded) & -3.13 & .038 \\
\hline & & V4 (25\% excluded) & -6.21 & .019 \\
\hline & & V4 (50\% excluded) & -3.57 & .021 \\
\hline & & IPS (5\% excluded) & -2.68 & .060 \\
\hline & & IPS (10\% excluded) & -2.48 & .076 \\
\hline & & IPS (25\% excluded) & -0.94 & .437 \\
\hline & & IPS (50\% excluded) & 0.20 & .862 \\
\hline
\end{tabular}

Supplemental Table 1. Statistics from tests depicted in Figure 2 from the main text. 


\section{Supplemental references.}

1. Ester, E. F., Sprague, T. C. \& Serences, J. T. Parietal and frontal cortex encode stimulus-specific mnemonic representations during visual working memory. Neuron 87, 893-905 (2015).

2. Ester, E. F., Anderson, D. E., Serences, J. T. \& Awh, E. A neural measure of precision in visual working memory. Journal of cognitive neuroscience $\mathbf{2 5}, \mathbf{7 5 4 - 7 6 1}$ (2013).

3. Foster, J. J., Bsales, E. M. \& Awh, E. Covert spatial attention speeds target individuation. Journal of Neuroscience 40, 2717-2726 (2020).

4. Henderson, M., Vo, V., Chunharas, C., Sprague, T. \& Serences, J. Multivariate analysis of BOLD activation patterns recovers graded depth representations in human visual and parietal cortex. Eneuro 6, (2019).

5. Kok, P., Rait, L. I. \& Turk-Browne, N. B. Content-based dissociation of hippocampal involvement in prediction. Journal of Cognitive Neuroscience 32, 527-545 (2020).

6. Liu, T., Cable, D. \& Gardner, J. L. Inverted encoding models of human population response conflate noise and neural tuning width. Journal of Neuroscience $\mathbf{3 8}, 398$ 408 (2018).

7. Oh, B.-I., Kim, Y.-J. \& Kang, M.-S. Ensemble representations reveal distinct neural coding of visual working memory. Nature communications 10, 1-12 (2019).

8. Yu, Q., Teng, C. \& Postle, B. R. Different states of priority recruit different neural representations in visual working memory. PLoS biology 18, e3000769 (2020). 
9. Rademaker, R. L., Chunharas, C. \& Serences, J. T. Coexisting representations of sensory and mnemonic information in human visual cortex. Nature neuroscience $\mathbf{2 2}$, 1336-1344 (2019).

10. Sprague, T. C. \& Serences, J. T. Attention modulates spatial priority maps in the human occipital, parietal and frontal cortices. Nature neuroscience 16, 1879-1887 (2013).

11. Sutterer, D. W., Foster, J. J., Adam, K. C., Vogel, E. K. \& Awh, E. Item-specific delay activity demonstrates concurrent storage of multiple active neural representations in working memory. PLoS biology 17, e3000239 (2019).

12. Cai, Y., Sheldon, A. D., Yu, Q. \& Postle, B. R. Overlapping and distinct contributions of stimulus location and of spatial context to nonspatial visual short-term memory. Journal of neurophysiology 121, 1222-1231 (2019).

13. Chen, N. et al. Sharpened cortical tuning and enhanced cortico-cortical communication contribute to the long-term neural mechanisms of visual motion perceptual learning. Neuroimage 115, 17-29 (2015).

14. Foster, J. J., Sutterer, D. W., Serences, J. T., Vogel, E. K. \& Awh, E. Alpha-band oscillations enable spatially and temporally resolved tracking of covert spatial attention. Psychological science 28, 929-941 (2017).

15. Garcia, J. O., Srinivasan, R. \& Serences, J. T. Near-real-time feature-selective modulations in human cortex. Current Biology 23, 515-522 (2013).

16. Ho, T. et al. The optimality of sensory processing during the speed-accuracy tradeoff. Journal of Neuroscience 32, 7992-8003 (2012). 
17. Kok, P. \& Turk-Browne, N. B. Associative prediction of visual shape in the hippocampus. Journal of Neuroscience 38, 6888-6899 (2018).

18. Lorenc, E. S., Sreenivasan, K. K., Nee, D. E., Vandenbroucke, A. R. \& D'Esposito, M. Flexible coding of visual working memory representations during distraction. Journal of Neuroscience 38, 5267-5276 (2018).

19. Mostert, P. et al. Eye movement-related confounds in neural decoding of visual working memory representations. Eneuro 5, (2018).

20. Samaha, J., Sprague, T. C. \& Postle, B. R. Decoding and reconstructing the focus of spatial attention from the topography of alpha-band oscillations. Journal of cognitive neuroscience 28, 1090-1097 (2016).

21. Sprague, T. C., Ester, E. F. \& Serences, J. T. Reconstructions of information in visual spatial working memory degrade with memory load. Current Biology 24, 2174-2180 (2014).

22. Sprague, T. C., Ester, E. F. \& Serences, J. T. Restoring latent visual working memory representations in human cortex. Neuron 91, 694-707 (2016).

23. Sprague, T. C., Itthipuripat, S., Vo, V. A. \& Serences, J. T. Dissociable signatures of visual salience and behavioral relevance across attentional priority maps in human cortex. Journal of neurophysiology 119, 2153-2165 (2018).

24. van Moorselaar, D. et al. Spatially selective alpha oscillations reveal moment-bymoment trade-offs between working memory and attention. Journal of cognitive neuroscience 30, 256-266 (2018). 
25. Vo, V. A., Sprague, T. C. \& Serences, J. T. Spatial tuning shifts increase the discriminability and fidelity of population codes in visual cortex. Journal of Neuroscience 37, 3386-3401 (2017).

26. Yu, Q. \& Shim, W. M. Temporal-order-based attentional priority modulates mnemonic representations in parietal and frontal cortices. Cerebral Cortex 29, 3182-3192 (2019).

27. Kok, P., Mostert, P. \& De Lange, F. P. Prior expectations induce prestimulus sensory templates. Proceedings of the National Academy of Sciences 114, 1047310478 (2017).

28. Tang, M. F., Arabzadeh, E. \& Mattingley, J. B. Forward modelling reveals dynamics of neural orientation tuning to unconscious visual stimuli during binocular rivalry. bioRxiv (2019) doi:10.1101/574905.

29. Kim, I., Hong, S. W., Shevell, S. K. \& Shim, W. M. Neural representations of perceptual color experience in the human ventral visual pathway. Proceedings of the National Academy of Sciences 117, 13145-13150 (2020).

30. Kok, P., Brouwer, G. J., van Gerven, M. A. \& de Lange, F. P. Prior expectations bias sensory representations in visual cortex. Journal of Neuroscience 33, 16275-16284 (2013). 